\title{
Clinical Verification and Some Observations on a Polychrest Homoeopathic Remedy Lachesis Muta in a Primary Health Center
}

\begin{abstract}
Introduction: Polychrest remedies like Lachesis muta are commonly found to be useful in both the acute and chronic morbid conditions. Between April 2012 and March 2013, more than 21000 patients visited the Delhi Government Homeopathic Dispensary Aali village, primary health centre, out of which nearly $1.4 \%$ cases presented with keynote symptomatology of Lachesis muta. It provided an ample opportunity to clinically verify some of the well-known indications of the polychrest.

Objectives: a. To clinically verify the indicating symptomatology of Lachesis muta in the study group. b. To observe, with respect to the polychrest remedy: The various clinical conditions in which it was prescribed; Posology; The minimum time of favorable response to the remedy in various clinical conditions; the mean time of recovery in each diagnostic condition, and; the therapeutic outcome.

Materials and Methods: A retrospective data analysis as per the objectives, of 193 patients confirming with the defined inclusion criteria was conducted.

Results and Discussion: These patients fell between 6 months and 70 years with 29.6 years being the mean age of presentation; out of these 67 were males and 126 were females. 25 characteristic features/concomitant symptoms or modalities of Lachesis muta from textbooks of homoeopathic materia medica were clinically verified and guided the polychrest remedy selection in 36 different diagnostic conditions. It was strongly verified in various acute conditions, that the response to indicated homoeopathic remedy can be judged within a few hours.

Conclusion: Though the homoeopathic materia medica by stalwarts is irreplaceable, their clinical sections require a scientific appraisal. This work is a first attempt to identify the clinical conditions amenable to a polychrest remedy in the current times and gauge the posology and remedy response time as well as therapeutic outcome. Further multi-centric studies with more scientific rigour can validate as well as clinically verify the symptomatology of Lachesis muta by the use of Bayesian statistics.
\end{abstract}

Keywords: Homoeopathic remedy; Lachesis muta; Data analysis; Symptomatology; Polychrests portray; Exclusion criteria; Bayesian statistics

Research Article
Volume 8 Issue 4 - 2017
Gyandas G Wadhwani*
Consultant Homoeopathic Physician \& Independent
Researcher, Holistic Homoeopathic Clinic \& Research Center,
India
*Corresponding author: Gyandas G Wadhwani, Consultant
Homoeopathic Physician \& Independent Researcher, Holistic
Homoeopathic Clinic \& Research Center, New Delhi, India.
Former Chief Medical Officer Directorate of Ayush, Govt of
National Capital Territory of Delhi, India,
Email: homoeopathygyan@gmail.com
Received: July 12, 2017 | Published: August 31, 2017

\section{Introduction}

Some medicines produce many symptoms on nearly the entire healthy human body during homeopathic drug proving (controlled human pathogenetic trials), which often correspond to the morbid symptoms commonly met with; hence they admit of frequent homoeopathic employment and often do good. Hahnemann termed these remedies of many uses, both in acute and chronic morbid conditions, as polychrest remedies [1].

These polychrests portray several archetypes, each of which embodies a wide range of symptoms and personality traits of which the individual patient is but a partial copy. Lachesis is one such polychrest remedy of homeopathic materia medica, which was introduced by Constantine Hering after proving the remedy upon himself and others. 'The Guiding Symptoms of Materia
Medica', reveals successful application of the remedy by various clinical authorities in nearly 175 diverse clinical conditions [2,3].

During the period between 1st April 2012 to 31st March 2013, more than 21000 patients visited the Delhi Government Homeopathic Dispensary Aali village (DGHDAV), primary health centre, who received their indicated homoeopathic remedy (including Lachesis muta) as per their individualizing totality.

The group of patients who received Lachesis muta presented with two of the most recognized signs, intolerance of constriction around neck and fine tremors in tongue. The former was identified in females, as the traditional Indian women (visiting DGHDAV) tend to wear some basic ornaments or use veil around neck, which was surprisingly missing. In the males, there was an uncommon frequency of visits to dispensaries wearing unbuttoned shirts 
(nearly top 2-3 buttons in all of the cases) and keeping collars pulled apart from the neck. In most of the cases in the study group, the loquacity was also well-marked objective symptom, without any regard to the diagnostic condition. The description of complaints or few more questions easily confirmed choice of the remedy.

It provided an ample opportunity to clinically verify some of the well-known indications of the polychrest remedy. The patients on the study group were further included for analysis (as per inclusion criteria) to ascertain its usage and response in terms of clinical conditions, therapeutic outcome, time for recovery.

\section{Objectives}

I. To clinically verify the indicating symptomatology of Lachesis muta in the study group

II. To observe with respect to Lachesis muta:

a. The various clinical conditions in which it was prescribed.

b. Posology.

c. The minimum time of favourable response to the remedy in various clinical conditions

d. The mean time of recovery in each diagnostic condition, and

e. The therapeutic outcome.

\section{Materials and methods}

During the study period between 01-04-2012 and 31-03-2013, 296 patients with different diseased conditions received lachesis as a first or second prescription at DGHDAV. A retrospective cross-sectional data analysis as per the objectives, of all the cases the cases that confirmed with the defined inclusion criteria was undertaken. Prior approval of the ethical committee was not taken since it was merely a retrospective data analysis.

\section{Study setting}

Aali Village is a rural area in the National Capital Territory of Delhi with a population of over 100,000 people, mostly migrants from various states of India, consisting of skilled labor and working class of low socio-economic group and their families with poor literacy rate. For them, each day of illness costs dearly on their meager sources of income as well as job opportunity/ availability. The DGHDAV has been providing primary health care services in the area since 1999.

The PHC functions with a basic staff of a single doctor and pharmacist. Since the patient turnout is high, the diagnosis and remedy selection is on the basis of objective signs (pathognomonic of disease or remedy) or accurate recording of the precisely described complaints. Since their tone and mannerisms are usually matter-of-fact and non-indulgent, nailing the diagnosis and selecting the remedy becomes comparatively simpler.

Due to poor financial status and absence of any government supported pathological laboratories nearby, serological/ radiological correlation cannot be conducted regularly.

\section{Inclusion/ exclusion criteria}

The following cases were included for data-analysis:

a. Presented with pre-defined symptomatology of lachesis, as available in the textbooks of homeopathic materia medica i.e. Constantine Hering's The Guiding Symptoms of our Materia Medica vol VI, Timothy Field Allen's The Encyclopedia of Pura Materia Medica vol V and John Henry Clarke's A Dictionary of Practical Materia Medica vol II [4-6].

b. Received lachesis as a first prescription at DGHDAV.

c. Lachesis was prescribed as a single homeopathic remedy, in accordance with homeopathic principles.

d. Followed up at least three times.

To minimise the effects of confounding variables, the patients were asked to discontinue the usage of all anti-inflammatory, anti-pyretic, antibiotics and analgesic remedies at the onset of treatment. Only the allopathic medications deemed necessary viz. anti-hypertensive, hypoglycemic drugs, were to be continued during the observational period. The usage of dietary supplements was also allowed only if necessary. Patients with a history of alcohol/drug abuse or under narcotic medication were excluded from the study.

\section{Frequency of remedy repetition, potency selection and dosage}

Only the centesimal potencies of the remedy were readily available at DGHDAV and all the patients received that. The remedy repetition and potency selection was in accordance with the guidelines of homeopathic philosophy [7]. Persons below 15 years of age received 2 globules and those above, received 4 globules as one dose.

\section{Assessment and follow-up}

All the cases were assessed between 2-14 days depending on the severity of complaints and convenience of the patient.

\section{Results}

Lachesis muta was prescribed in 296 out of 21000 patients (1.4\%) who visited DGHDAV between 1st April 2012 and 31st March 2013.

While $46.63 \%$ patients visited the PHC for treatment as first line of choice, the other patients patients called upon after failure/ unsatisfactory response after previous treatment (53.37\%) Allopathic (48.19\%), Homoeopathy (0.52\%), Ayurveda (3.63\%), Others (1.03\%). 193 patients were taken up for retrospective data analysis as per the inclusion criteria (Figures 1-2), (Tables 1-2).

Lachesis was prescribed in 36 diverse clinical conditions both acute and chronic [Table 3]. This verifies the polychrest nature of the remedy.

30, 200 and 1000 potencies of Lachesis muta were administered, in ascending order, as per the need of the case, in all the treated clinical conditions and, their repetition (posology) may be seen in Table 4. Minimum mean time for gauging positive remedy response in each patient, along with average recovery 
time and the therapeutic outcome may also be seen below.

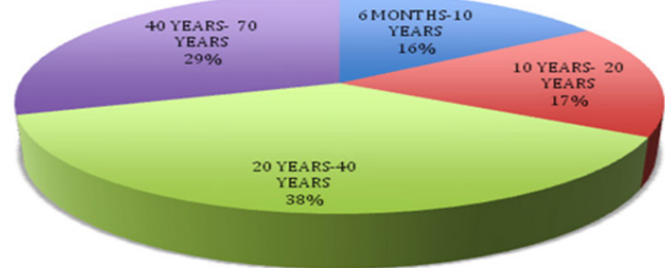

Figure 1: Age-wise percentages of patients who received Lachesis muta.

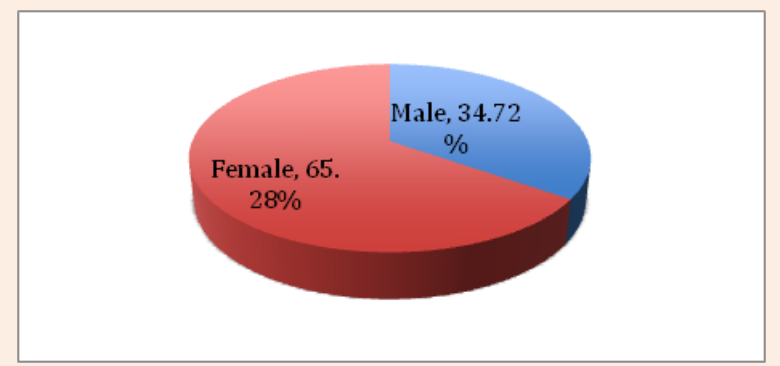

Figure 2: Gender distribution of patients receiving lachesis.

Table 1: Clinical verification

\begin{tabular}{|c|c|c|c|c|c|c|c|c|c|}
\hline S.No & $\begin{array}{c}\text { Indicative } \\
\text { Symptomatology }\end{array}$ & $\begin{array}{l}\text { Objective } \\
\text { (0)/ } \\
\text { Subjective } \\
\text { (S) }\end{array}$ & $\begin{array}{l}\text { Number } \\
\text { of } \\
\text { Patients }\end{array}$ & Percentage & Remarks & $\begin{array}{l}\text { Hering's } \\
\text { Guiding } \\
\text { Symptoms }\end{array}$ & $\begin{array}{c}\text { Allen's } \\
\text { Encyclopedia }\end{array}$ & $\begin{array}{l}\text { Clarke's } \\
\text { Dictionary }\end{array}$ & $\begin{array}{l}\text { Henry Clay } \\
\text { Allen's } \\
\text { Keynotes(8) }\end{array}$ \\
\hline 1. & Insane jealousy & 0 & 70 & $36.27 \%$ & \multirow[t]{2}{*}{$\begin{array}{l}\text { Mostly discerned } \\
\text { in children and } \\
\text { adolescents }\end{array}$} & $\checkmark$ & $\checkmark$ & $\checkmark$ & $\begin{array}{l}\checkmark \text { (Ailments } \\
\text { from jealousy; } \\
\text { Epilepsy from } \\
\text { jealousy) }\end{array}$ \\
\hline 2. & Suspiciousness & 0 & 62 & $32.12 \%$ & & $\checkmark$ & $\checkmark$ (Mistrust) & $\checkmark$ & - \\
\hline 3. & $\begin{array}{c}\text { Talkativeness/ } \\
\text { loquacity }\end{array}$ & 0 & 154 & $79.79 \%$ & & $\checkmark$ & $\checkmark$ & $\checkmark$ & $\checkmark$ \\
\hline 4. & Religious & 0 & 72 & $37.31 \%$ & & $\checkmark$ & $\checkmark$ & $\begin{array}{l}\checkmark(\text { Desire to } \\
\text { meditate })\end{array}$ & - \\
\hline 5. & Suffocation & $\mathrm{s}$ & 161 & $83.42 \%$ & $\begin{array}{l}\text { In the absence of any } \\
\text { pathological reason, } \\
\text { they are prone to } \\
\text { sensation of suffocation. } \\
\text { For example in a warm } \\
\text { place (hence may need } \\
\text { doors and windows } \\
\text { open, but this is not } \\
\text { typical claustrophobia), } \\
\text { while pulling down a } \\
\text { pullover, can't sleep } \\
\text { facing the wall (shall } \\
\text { face the open area) etc. }\end{array}$ & $\checkmark$ & $\checkmark$ & $\checkmark$ & $\checkmark$ \\
\hline 6. & $\begin{array}{l}\text { Intolerance of } \\
\text { constriction } \\
\text { around neck }\end{array}$ & 0 & 193 & $100 \%$ & $\begin{array}{l}\text { Could not wear any } \\
\text { clothing with collar/ } \\
\text { high neck/ jewellery } \\
\text { pieces/ neck-ties } \\
\text { around neck etc. }\end{array}$ & $\checkmark$ & $\checkmark$ & $\checkmark$ & $\checkmark$ \\
\hline 7. & $\begin{array}{l}\text { Intolerance } \\
\text { of tight fitting } \\
\text { garments }\end{array}$ & $0 / \mathrm{S}$ & 187 & $96.89 \%$ & $\begin{array}{l}\text { These patients could } \\
\text { not tolerate any ring, } \\
\text { bracelets/ bangles, } \\
\text { belts etc. }\end{array}$ & $\checkmark$ & - & $\checkmark$ & $\checkmark$ \\
\hline 8. & $\begin{array}{l}\text { Aggravation of } \\
\text { complaints while } \\
\text { falling asleep }\end{array}$ & $\mathrm{s}$ & 135 & $69.95 \%$ & $\begin{array}{l}\text { In almost all these } \\
\text { patients the aggravation } \\
\text { of complaints was felt } \\
\text { while the patient was } \\
\text { about to fall asleep i.e. } \\
\text { they would wake up } \\
\text { soon after falling asleep } \\
\text { (within a few minutes) }\end{array}$ & $\checkmark$ & $\checkmark$ & $\checkmark$ & $\checkmark$ \\
\hline
\end{tabular}

Citation: Wadhwani GG (2017) Clinical Verification and Some Observations on a Polychrest Homoeopathic Remedy Lachesis Muta in a Primary Health Center. Int J Complement Alt Med 8(4): 00269. DOI: 10.15406/ijcam.2017.08.00269 


\begin{tabular}{|c|c|c|c|c|c|c|c|c|c|}
\hline 9. & Left sided & $\mathrm{S} / 0$ & 86 & $44.56 \%$ & \multirow{2}{*}{ S- sensations; 0- lesions } & $\checkmark$ & $\checkmark$ & $\checkmark$ & $\checkmark$ \\
\hline 10. & Left to right & $\mathrm{S} / 0$ & 81 & $41.97 \%$ & & - & $\checkmark$ & $\checkmark$ & $\checkmark$ \\
\hline 11. & $\begin{array}{l}\text { Bluish } \\
\text { discoloration } \\
\text { around mouth }\end{array}$ & 0 & 89 & $46.11 \%$ & $\begin{array}{l}\text { A faint bluish peri-oral } \\
\text { discoloration could } \\
\text { be seen }\end{array}$ & $\begin{array}{l}\text { TI (Bluish } \\
\text { dis- } \\
\text { oloration/ } \\
\text { spots/ hue } \\
\text { on face) }\end{array}$ & $\checkmark$ & $\checkmark$ & - \\
\hline 12. & $\begin{array}{l}\text { Bluish } \\
\text { discoloration }\end{array}$ & 0 & 6 & $3.11 \%$ & Of skin lesions & $\checkmark$ & $\checkmark$ & $\checkmark$ & $\checkmark$ \\
\hline 13. & $\begin{array}{l}\text { Tongue: difficult } \\
\text { to protrude }\end{array}$ & 0 & 98 & $50.78 \%$ & $\begin{array}{l}\text { A lot of patients would } \\
\text { protrude the tongue } \\
\text { with a jerk or could } \\
\text { not keep it protruded } \\
\text { for long. }\end{array}$ & $\checkmark$ & $\checkmark$ & $\checkmark$ & $\checkmark$ \\
\hline 14. & $\begin{array}{l}\text { Tongue: catches } \\
\text { in teeth }\end{array}$ & 0 & 87 & $45.08 \%$ & $\begin{array}{l}\text { These patients also } \\
\text { could not protrude } \\
\text { tongue easily as it } \\
\text { would get caught in } \\
\text { teeth. }\end{array}$ & $\checkmark$ & $\checkmark$ & $\checkmark$ & $\checkmark$ \\
\hline 15. & $\begin{array}{l}\text { Tongue: fine } \\
\text { tremors }\end{array}$ & 0 & 193 & $100 \%$ & $\begin{array}{l}\text { The characteristic } \\
\text { tremors were fine } \\
\text { tremors not coarse }\end{array}$ & $\checkmark$ & $\checkmark$ & $\checkmark$ & $\checkmark$ \\
\hline 16. & $\begin{array}{l}\text { Complaints } \\
\text { improve } \\
\text { with onset of } \\
\text { menstruation }\end{array}$ & s & 30 & $15.54 \%$ & $\begin{array}{l}\text { In most of these } \\
\text { patients the } \\
\text { symptomatolo-gy } \\
\text { improved as the } \\
\text { menstrual flow } \\
\text { increased }\end{array}$ & $\checkmark$ & $\checkmark$ & $\checkmark$ & $\checkmark$ \\
\hline 17. & $\begin{array}{l}\text { Menses at regular } \\
\text { time }\end{array}$ & $\mathrm{s}$ & 8 & $4.15 \%$ & $\begin{array}{l}\text { These patients had } \\
\text { a clock like regular } \\
\text { menstrual cycle, like } \\
\text { every } 28 \text { or } 30 \text { days. }\end{array}$ & $\checkmark$ & & $\checkmark$ & $\checkmark$ \\
\hline 18. & $\begin{array}{c}\text { Swallowing } \\
\text { liquids aggravates }\end{array}$ & $\mathrm{s}$ & 8 & $4.15 \%$ & \multirow{3}{*}{ Seen in acute cases only. } & $\checkmark$ & $\checkmark$ & $\checkmark$ & $\checkmark$ \\
\hline 19. & $\begin{array}{l}\text { Swallowing empty } \\
\text { aggravates }\end{array}$ & $\mathrm{s}$ & 6 & $3.11 \%$ & & $\checkmark$ & $\checkmark$ & $\checkmark$ & - \\
\hline 20. & $\begin{array}{l}\text { Inclination to } \\
\text { swallow }\end{array}$ & 0 & 12 & $6.22 \%$ & & $\checkmark$ & $\checkmark$ & $\checkmark$ & - \\
\hline 21. & $\begin{array}{l}\text { Desire to take } \\
\text { a deep breath/ } \\
\text { involuntary } \\
\text { sighing }\end{array}$ & 0 & 15 & $7.77 \%$ & $\begin{array}{l}\text { In the absence of any } \\
\text { respiratory difficulty. }\end{array}$ & $\checkmark$ & $\checkmark$ & $\checkmark$ & $\begin{array}{l}\checkmark \text { (Mentioned } \\
\text { under Ignatia) }\end{array}$ \\
\hline 22. & $\begin{array}{l}\text { Ailments during } \\
\text { spring weather }\end{array}$ & $0 / \mathrm{S}$ & 23 & $11.92 \%$ & & $\checkmark$ & $\checkmark$ & $\checkmark$ & $\checkmark$ \\
\hline 23. & $\begin{array}{c}\text { Thermal reaction: } \\
\text { hot }\end{array}$ & s & 107 & $55.44 \%$ & \multirow{3}{*}{$\begin{array}{l}\text { Though primarily a } \\
\text { 'hot' polychrest remedy, } \\
\text { patients requiring this } \\
\text { remedy may be chilly } \\
\text { or sensitive to extreme } \\
\text { temperature too. Even } \\
\text { when chilly they can't } \\
\text { bear over-clothing/ } \\
\text { over-covering and feel } \\
\text { suffocated. }\end{array}$} & $\checkmark$ & $\checkmark$ & $\checkmark$ & $\checkmark$ \\
\hline 24. & $\begin{array}{l}\text { Thermal reaction: } \\
\text { chilly }\end{array}$ & $\mathrm{s}$ & 33 & $17.1 \%$ & & $\checkmark$ & - & $\checkmark$ & - \\
\hline 25. & $\begin{array}{l}\text { Thermal reaction: } \\
\text { sensitive to } \\
\text { extremes of heat/ } \\
\text { cold }\end{array}$ & $\mathrm{s}$ & 53 & $27.46 \%$ & & $\checkmark$ & $\checkmark$ & - & $\checkmark$ \\
\hline
\end{tabular}

The following symptoms (Table 2), though not mentioned in textbooks of materia medica, were also clinically verified in the presence of other characteristic features of remedy.

In accordance with the first objective, twenty-eight signs and symptoms of Lachesis muta, mentioned in the textbooks of homoeopathic materia medica could be clinically verified (Tables 1 \& 2). 
Table 2: Other symptoms of Lachesis muta that were clinically verified.

\begin{tabular}{|c|c|c|c|c|c|c|}
\hline S. No & $\begin{array}{c}\text { Indicative } \\
\text { Symptomatology }\end{array}$ & $\begin{array}{l}\text { Objective (0)/ } \\
\text { Subjective (S) }\end{array}$ & $\begin{array}{l}\text { Number of } \\
\text { Patients }\end{array}$ & Percentage & Remarks & Verification from Old Text books \\
\hline 1. & Sibling rivalry* & 0 & 69 & $35.75 \%$ & $\begin{array}{l}\text { Mostly discerned } \\
\text { in children and } \\
\text { adolescents }\end{array}$ & $\begin{array}{c}\text { Though not mentioned in any } \\
\text { textbook of Materia Medica, but in } \\
\text { the background of jealousy, verified } \\
\text { in numerous cases when other } \\
\text { symptoms of lachesis were also } \\
\text { present. }\end{array}$ \\
\hline 2. & Fear of snakes & S & 34 & $17.62 \%$ & $\begin{array}{l}\text { Six of these patients } \\
\text { dated these fears to } \\
\text { unexpectedly coming } \\
\text { across a snake at their } \\
\text { village. None of the } \\
\text { others could correlate } \\
\text { their fear of snake with } \\
\text { any past experience. }\end{array}$ & $\begin{array}{l}\text { Hering, in his Guiding symptoms, } \\
\text { mentions the case of a } 9 \text { years old } \\
\text { girl frightened by snake [9]. Out of } 34 \\
\text { patients only } 6 \text { could correlate this } \\
\text { fear to some happening in their lives. } \\
\text { The remedy is mentioned under this } \\
\text { rubric of Synthetic Repertory and } \\
\text { attributed to Pierre Schmidt [10] }\end{array}$ \\
\hline 3. & Dreams of snakes & S & 24 & $12.44 \%$ & $\begin{array}{l}\text { Out of these } 24 \text { nearly } \\
10 \text { also had a strong } \\
\text { fear of snakes. In } 3 \text { male } \\
\text { and } 2 \text { female patients } \\
\text { these dreams were } \\
\text { sexual in nature. }\end{array}$ & $\begin{array}{c}\text { Mentioned under rubric of Synthetic } \\
\text { Repertory and attributed to Pierre } \\
\text { Schmidt [11] }\end{array}$ \\
\hline
\end{tabular}

*Though not mentioned in any textbook of Materia Medica, but in the background of jealousy, verified in numerous cases when other characteristic symptoms of lachesis were also present.

Table 3: Various clinical conditions in which polychrest were prescribed at PHC.

\begin{tabular}{|c|c|c|c|c|}
\hline S. No. & Name of Disease/ Diagnosis & No. of Patients & Male; Females & Percentage \\
\hline 1. & Bronchial asthma & 12 & $4 ; 8$ & $6.22 \%$ \\
\hline 2. & Migraine & 4 & $0 ; 4$ & $2.07 \%$ \\
\hline 3. & Upper respiratory tract infection with fever & 10 & $4 ; 6$ & $5.18 \%$ \\
\hline 4. & Non-specific cervical lymphadenitis (in children) & 4 & $1 ; 3$ & $2.07 \%$ \\
\hline 5. & Warts & 3 & $1 ; 2$ & $1.55 \%$ \\
\hline 6. & Tinea capitis & 6 & $2 ; 4$ & $3.11 \%$ \\
\hline 7. & Tinea cruris & 4 & $3 ; 1$ & $2.07 \%$ \\
\hline 8. & Vitiligo & 5 & $2 ; 3$ & $2.59 \%$ \\
\hline 9. & Acne vulgaris & 9 & $4 ; 5$ & $4.66 \%$ \\
\hline 10. & Seborrhoeic dermatitis & 8 & $2 ; 6$ & $4.15 \%$ \\
\hline 11. & Amoebic colitis & 2 & $1 ; 1$ & $1.04 \%$ \\
\hline 12. & Irritable bowel syndrome & 2 & $2 ; 0$ & $1.04 \%$ \\
\hline 13. & Hypothyroidism & 3 & $0 ; 3$ & $1.55 \%$ \\
\hline 14. & Diabetes mellitus & 2 & $0 ; 2$ & $1.04 \%$ \\
\hline 15. & Hypertension & 4 & $2 ; 2$ & $2.07 \%$ \\
\hline 16. & Rheumatoid arthritis & 9 & $1 ; 8$ & $4.66 \%$ \\
\hline 17. & Alopecia areata & 5 & $3 ; 2$ & $2.59 \%$ \\
\hline 18. & Molluscum contagiosum & 6 & $3 ; 3$ & $3.11 \%$ \\
\hline
\end{tabular}




\begin{tabular}{|c|c|c|c|c|}
\hline 19. & COPD & 8 & $5 ; 3$ & $4.15 \%$ \\
\hline 20. & LRTI with fever & 6 & $3 ; 3$ & $3.11 \%$ \\
\hline 21. & Ovarian cyst & 4 & $0 ; 4$ & $2.07 \%$ \\
\hline 22. & Uterine fibroids & 5 & $0 ; 5$ & $2.59 \%$ \\
\hline 23. & DUB & 9 & $0 ; 9$ & $4.66 \%$ \\
\hline 24. & ASOM & 10 & $5 ; 5$ & $5.18 \%$ \\
\hline 25. & Glue ear & 9 & $5 ; 4$ & $4.66 \%$ \\
\hline 26. & CSOM & 4 & $2 ; 2$ & $2.07 \%$ \\
\hline 27. & Thyrotoxicosis & 2 & $0 ; 2$ & $1.04 \%$ \\
\hline 28. & Melasma & 9 & $1 ; 8$ & $4.66 \%$ \\
\hline 29. & Carpal tunnel syndrome & 4 & $1 ; 3$ & $2.07 \%$ \\
\hline 30. & Dengue fever & 5 & $3 ; 2$ & $2.59 \%$ \\
\hline 31. & Chikungunya fever & 3 & $2 ; 1$ & $1.55 \%$ \\
\hline 32. & Post chikungunya chronic arthritis & 1 & $0 ; 1$ & $0.52 \%$ \\
\hline 33. & Anal fissure & 6 & $2 ; 4$ & $3.11 \%$ \\
\hline 34. & Hemorrhoids & 4 & $2 ; 2$ & $2.07 \%$ \\
\hline 35. & Psoriasis & 5 & $1 ; 4$ & $2.59 \%$ \\
\hline 36. & Epidermolysis bullosa & 1 & $0 ; 1$ & $0.52 \%$ \\
\hline
\end{tabular}

Table 4: Posology, remedial action and therapeutic outcome.

\begin{tabular}{|c|c|c|c|c|c|}
\hline S. No. & $\begin{array}{c}\text { Name of } \\
\text { Disease/ } \\
\text { Diagnosis }\end{array}$ & $\begin{array}{l}\text { Potency } \\
\text { Prescribed }\end{array}$ & Repetition & $\begin{array}{l}\text { Time of } \\
\text { Response of } \\
\text { Remedy }\end{array}$ & Therapeutic Response \\
\hline 1. & $\begin{array}{l}\text { Bronchial } \\
\text { asthma }\end{array}$ & 30,200 & $\begin{array}{l}\text { Three doses: } \\
\text { each to be taken } \\
\text { every } 4 \text { hours } \\
\text { if the patient } \\
\text { consulted } \\
\text { during acute } \\
\text { phase or every } \\
12 \text { hours during } \\
\text { remission }\end{array}$ & $\begin{array}{l}30 \text { min-5 hours } \\
\text { during acute } \\
\text { phase. } \\
\\
\text { 3-6 weeks to } \\
\text { subjectively feel } \\
\text { improvement in } \\
\text { chronic cases. }\end{array}$ & $\begin{array}{l}\text { Four acute cases responded very well and could be managed } \\
\text { without supportive measures at the PHC. } \\
\text { The } 8 \text { chronic cases are still under follow-up: } \\
\text { - Two cases faced no acute episode over six-eight } \\
\text { months } \\
\text { - Five cases faced minor episodes which could be } \\
\text { managed with placebo and reported decreased } \\
\text { sensitivity to triggers. } \\
\text { - One case dropped out after three months follow-up } \\
\text { due to job transfer. He also reported improvement. }\end{array}$ \\
\hline 2. & Migraine & $\begin{array}{l}30,200 \\
1000\end{array}$ & $\begin{array}{l}\text { Three doses: } \\
\text { each to be taken } \\
\text { every } 4 \text { hours } \\
\text { if the patient } \\
\text { consulted } \\
\text { during acute } \\
\text { phase or every } \\
12 \text { hours during } \\
\text { remission }\end{array}$ & $\begin{array}{l}\text { 2-6 hours during } \\
\text { acute phase. }\end{array}$ & $\begin{array}{l}\text { - One lady consulted during acute phase and } \\
\text { recovered without any analgesic. She did not face } \\
\text { any episode of migraine during next } 5 \text { months } \\
\text { follow-up. } \\
\text { - Three other ladies consulted during remission and } \\
\text { were still being followed-up at the conclusion } \\
\text { o Two cases faced no episode over last four months. } \\
\text { o } \quad \text { One reported decreased sensitivity to triggers and } \\
\text { frequency. }\end{array}$ \\
\hline
\end{tabular}




\begin{tabular}{|c|c|c|c|c|c|}
\hline 3. & $\begin{array}{l}\text { Upper } \\
\text { respiratory tract } \\
\text { infection with } \\
\text { fever }\end{array}$ & 30,200 & $\begin{array}{l}\text { Three doses: } \\
\text { each to be taken } \\
\text { every } 3 \text { hours }\end{array}$ & $\begin{array}{l}\text { The best sign of } \\
\text { action of remedy } \\
\text { was undisturbed } \\
\text { sleep; it took } \\
4-6 \text { hours for } \\
\text { the fever to start } \\
\text { subsiding. }\end{array}$ & Complete recovery within 2-4 days in all cases. \\
\hline 4. & $\begin{array}{l}\text { Non-specific } \\
\text { cervical } \\
\text { lymphadenitis } \\
\text { (in children) }\end{array}$ & 200,1000 & $\begin{array}{l}\text { Three doses: } \\
\text { each to be taken } \\
\text { every } 12 \text { hours }\end{array}$ & 7-14 days & Complete recovery in all cases within $12-44$ days \\
\hline 5. & Warts & 200 & $\begin{array}{l}\text { Three doses: } \\
\text { each to be taken } \\
\text { every } 12 \text { hours }\end{array}$ & 14 days- 1 month & $\begin{array}{l}\text { Complete recovery without any topical application was seen } \\
\text { in all three cases within 6-8 weeks. }\end{array}$ \\
\hline 6. & Tinea capitis & 200 & $\begin{array}{l}\text { Three doses: } \\
\text { each to be taken } \\
\text { every } 12 \text { hours }\end{array}$ & $\begin{array}{l}14 \text { days to } 1 \\
\text { month }\end{array}$ & $\begin{array}{l}\text { - Complete recovery without any topical application } \\
\text { was seen in four cases within 6-8 weeks. } \\
\text { - Two cases showing improvement dropped out of } \\
\text { treatment as they went back to their native villages } \\
\text { after } 3 \text { follow-ups }\end{array}$ \\
\hline 7. & Tinea cruris & 200 & $\begin{array}{l}\text { Three doses: } \\
\text { each to be taken } \\
\text { every } 12 \text { hours }\end{array}$ & $\begin{array}{l}14 \text { days to } 1 \\
\text { month }\end{array}$ & $\begin{array}{l}\text { - Complete recovery without any topical application } \\
\text { was seen in two cases in } 3 \& 5 \text { months respectively. } \\
\text { - Two cases showing improvement dropped out of } \\
\text { treatment as they went back to their native villages } \\
\text { after } 4 \& 5 \text { follow-ups }\end{array}$ \\
\hline 8. & Vitiligo & 200 & $\begin{array}{l}\text { One dose given } \\
\text { stat }\end{array}$ & 4-5 weeks & $\begin{array}{l}\text { - Complete re-pigmentation in a case of focal vitiligo } \\
\text { of hands in } 4 \text { months } \\
\text { - Partial re-pigmentation observed in two cases } \\
\text { without new spots } \\
\text { - Two cases dropped out after } 4-5 \text { follow-ups citing } \\
\text { slow improvement }\end{array}$ \\
\hline 9. & Acne vulgaris & 200 & One dose stat & 2-4 weeks & $\begin{array}{l}\text { - Complete recovery in four cases in 4-5 months } \\
\text { - Marked reduction in number and frequency of } \\
\text { eruptions in two cases within 4-5 months } \\
\text { - Three cases dropped out after 4-5 follow-ups citing } \\
\text { slow improvement }\end{array}$ \\
\hline 10. & $\begin{array}{l}\text { Seborrhoeic } \\
\text { dermatitis }\end{array}$ & 200 & One dose stat & 4-6 weeks & $\begin{array}{l}\text { - Marked improvement in four cases in 6-8 months of } \\
\text { follow-up with reduction in scalp/ face oiliness and } \\
\text { frequency of eruptions } \\
\text { - Four cases dropped out after 4-5 follow-ups citing } \\
\text { slow improvement }\end{array}$ \\
\hline 11. & Amoebic colitis & $\begin{array}{l}30 \text { followed } \\
\text { by } 200\end{array}$ & $\begin{array}{c}\text { Three doses } \\
\text { of } 30 \text { potency } \\
\text { during acute } \\
\text { phase, } 12 \\
\text { hourly; after } \\
\text { recovery } 3 \\
\text { doses of } 200 \\
\text { potency, } 12 \\
\text { hourly }\end{array}$ & 4 hours & $\begin{array}{c}\text { Complete recovery in both the cases. Since both were chronic } \\
\text { patients having relapse of diarrhoea with griping \& bleeding } \\
\text { every 2-3 months, no further complaints were seen during } 8 \\
\text { months of follow-up. }\end{array}$ \\
\hline
\end{tabular}




\begin{tabular}{|c|c|c|c|c|c|}
\hline 12. & $\begin{array}{l}\text { Irritable bowel } \\
\text { syndrome }\end{array}$ & 200 & $\begin{array}{l}\text { Three doses: } \\
\text { each to be taken } \\
\text { every } 12 \text { hours }\end{array}$ & -- & $\begin{array}{l}\text { - One case showing improvement dropped out of } \\
\text { treatment as he went back to his native village after } \\
4 \& 5 \text { follow-ups } \\
\text { - One case dropped out after } 4-5 \text { follow-ups, citing } \\
\text { slow improvement }\end{array}$ \\
\hline 13. & Hypothyroidism & 200,1000 & $\begin{array}{c}\text { One dose stat } \\
\text { of } 200 \text { in two } \\
\text { cases; third } \\
\text { case required } \\
\text { a dose of } 1000 \\
\text { potency after } 5 \\
\text { months }\end{array}$ & 4-8 weeks & $\begin{array}{l}\text { One lady, clinically euthyroid, presented with TSH } \\
\text { 5.67; repeat TSH levels after 4, } 8 \text { and } 12 \text { weeks } \\
\text { were }<4 \\
\text { - } \quad \text { One lady with menorrhagia and TSH } 8.67 \text { recovered } \\
\text { in } 3 \text { months; TSH levels stayed }<4 \text { in repeat tests at } \\
5 \text { and } 7 \text { months } \\
\text { - } \quad \text { One lady is taking reduced dosage of Eltroxin from } \\
125 \text { mcg to } 50 \text { and } 75 \text { mcg alternate day after } 11 \\
\text { months of follow-up }\end{array}$ \\
\hline 14. & $\begin{array}{l}\text { Diabetes } \\
\text { mellitus }\end{array}$ & 200 & $\begin{array}{l}\text { Three doses: } \\
\text { each to be taken } \\
\text { every } 12 \text { hours }\end{array}$ & -- & $\begin{array}{l}\text { Both the cases had symptomatic improvement in weakness, } \\
\text { episodes of diarrhoea, symptoms of peripheral neuropathy } \\
\text { and skin complaints. No reduction in usage of hypoglycaemic } \\
\text { drugs during } 9 \& 10 \text { months of follow-up }\end{array}$ \\
\hline 15. & Hypertension & 200 & $\begin{array}{l}\text { Three doses: } \\
\text { each to be taken } \\
\text { every } 12 \text { hours }\end{array}$ & -- & $\begin{array}{l}\text { All the cases were followed up for 6-11 months: } \\
\text { - Two cases had symptomatic improvement in } \\
\text { frequency \& episodes of headache and dizziness. } \\
\text { No reduction in dosage of anti-hypertensive } \\
\text { medications. } \\
\text { - One case had no further episode of headache } \\
\text { with epistaxis during } 8 \text { moths of follow-up } \\
\text { (pre-treatment: } 2 \text { episodes every } 3 \text { months). } \\
\text { No reduction in dosage of anti-hypertensive } \\
\text { medications. } \\
\text { One case had symptomatic improvement in } \\
\text { frequency \& episodes of headache and vertigo; } \\
\text { insomnia improved considerably. No reduction in } \\
\text { dosage of anti-hypertensive medications. After } 6 \\
\text { months repeated blood tests showed Triglycerides } \\
\text { decreased from } 415 \text { to 275; Cholesterol from 255 } \\
\text { to 210; LDL from } 199 \text { to } 127 \text {; VLDL from } 49 \text { to 33; } \\
\text { HDL increased from } 35 \text { to } 42 \text {. }\end{array}$ \\
\hline 16. & $\begin{array}{l}\text { Rheumatoid } \\
\text { arthritis }\end{array}$ & $\begin{array}{c}30,200 \\
1000\end{array}$ & $\begin{array}{l}\text { Three doses } \\
\text { of any potency } \\
\text { prescribed: } \\
\text { each to be taken } \\
\text { every } 12 \text { hours }\end{array}$ & 2-4 weeks & 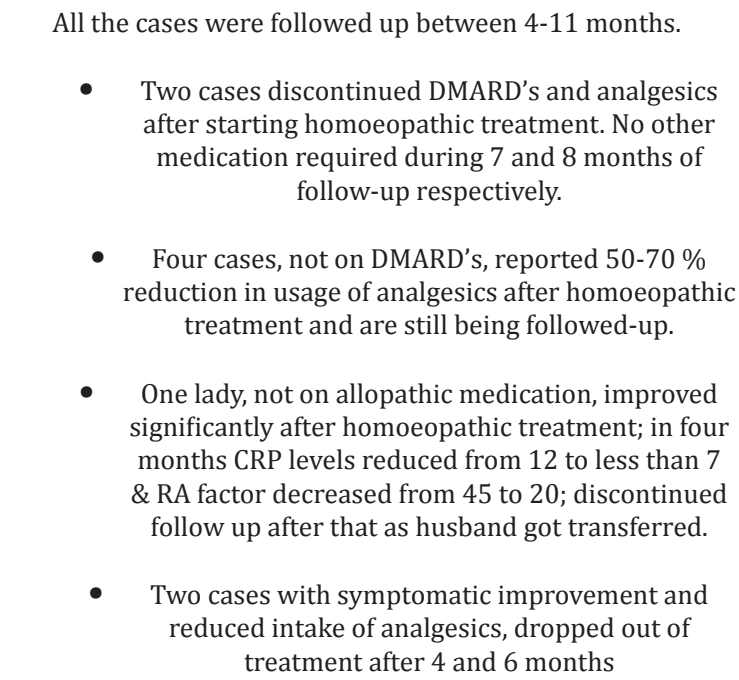 \\
\hline
\end{tabular}




\begin{tabular}{|c|c|c|c|c|c|}
\hline 17. & Alopecia areata & 200 & One dose stat & 2-3 weeks & $\begin{array}{l}\text { Three cases recovered within } 2 \text { months. } \\
\text { - One case required a repeat dose of } 200 \text { potency } \\
\text { after two months and recovered in } 4 \text { months. }\end{array}$ \\
\hline 18. & $\begin{array}{l}\text { Molluscum } \\
\text { contagiosum }\end{array}$ & 200 & One dose stat & 2-3 weeks & $\begin{array}{l}\text { - } \quad \text { Four cases recovered within } 2 \text { months with single } \\
\text { dose. } \\
\text { - Two cases required a dose of } 200 \text { potency after } \\
\text { every month and recovered in } 4 \text { months. }\end{array}$ \\
\hline 19. & COPD & $\begin{array}{c}30,200 \\
1000\end{array}$ & $\begin{array}{l}\text { Three doses } \\
\text { of any potency } \\
\text { prescribed: } \\
\text { each to be taken } \\
\text { every } 12 \text { hours }\end{array}$ & 2 weeks ??? & $\begin{array}{l}\text { All the cases were followed up between 6-11 months: } \\
\text { - Five cases reported marked improvement and a } \\
\text { comfortable winter without any trouble; no other } \\
\text { medication required. } \\
\text { - Two cases reported marked improvement and } \\
\text { dropped out of treatment after going back to village } \\
\text { mid-winter } \\
\text { - One case required allopathic treatment during } \\
\text { winter twice as he refused to quit smoking (two } \\
\text { courses of antibiotics and nebulisation) }\end{array}$ \\
\hline 20. & LRTI with fever & 30,200 & $\begin{array}{l}\text { Three doses } \\
\text { of any potency } \\
\text { prescribed: } \\
\text { each to be taken } \\
\text { every } 12 \text { hours }\end{array}$ & 12-24 hours & $\begin{array}{l}\text { All six cases recovered within } 5 \text { days of treatment. } \\
\text { All the cases were prescribed three doses of } 30^{\text {th }} \text { potency } \\
\text { initially. } \\
\text { - Two cases recovered without further repetition of } \\
\text { medicine } \\
\text { - Four cases were prescribed } 200^{\text {th }} \text { potency if fever } \\
\text { persisted on } 3^{\text {rd }} \text { day too. }\end{array}$ \\
\hline 21. & Ovarian cyst & 200,1000 & $\begin{array}{l}\text { Three doses } \\
\text { of any potency } \\
\text { prescribed: } \\
\text { each to be taken } \\
\text { every } 12 \text { hours } \\
\text { (not during or } \\
\text { 1week prior to } \\
\text { menses) }\end{array}$ & $\begin{array}{l}\text { Symptomatic } \\
\text { improvement } \\
\text { noticed in } \\
\text { the following } \\
\text { menstrual period }\end{array}$ & $\begin{array}{l}\text { The cyst sizes were between } 15-40 \mathrm{ml} \text {. All the cases improved } \\
\text { symptomatically. } \\
\text { - Only one lady repeated ultrasound } 5 \text { months she } \\
\text { received a single dose of lachesis } 200 \text { (symptomatic } \\
\text { relief was reported in the following menstrual } \\
\text { cycle). Her left sided hemorrhagic ovarian cyst } \\
\text { disappeared. } \\
\text { Three patients with left sided cysts refused repeat } \\
\text { ultrasound citing financial inability in the absence } \\
\text { of any physical complaint }\end{array}$ \\
\hline 22. & Uterine fibroids & $\begin{array}{c}30,200 \\
1000\end{array}$ & $\begin{array}{l}\text { Three doses } \\
\text { of any potency } \\
\text { prescribed: } \\
\text { each to be taken } \\
\text { every } 12 \text { hours } \\
\text { (not during or } \\
\text { 1week prior to } \\
\text { menses) }\end{array}$ & -- & $\begin{array}{l}\text { All cases presented with multiple uterine fibroids with sizes } \\
\text { varying between seedling and } 12 \times 10 \text { mm (maximum). } \\
\text { - Three cases followed up for 5,7 \& } 8 \text { months } \\
\text { respectively. All of them reported decreased } \\
\text { bleeding and pain. None of them repeated } \\
\text { ultrasound. } \\
\text { - Two cases dropped out of treatment after two } \\
\text { menstrual cycles as they felt no relief in pain and } \\
\text { bleeding. }\end{array}$ \\
\hline
\end{tabular}




\begin{tabular}{|c|c|c|c|c|c|}
\hline 23. & DUB & 200,1000 & $\begin{array}{l}\text { Three doses } \\
\text { of any potency } \\
\text { prescribed: } \\
\text { each to be taken } \\
\text { every } 12 \text { hours } \\
\text { (not during or } \\
\text { 1week prior to } \\
\text { menses) }\end{array}$ & -- & 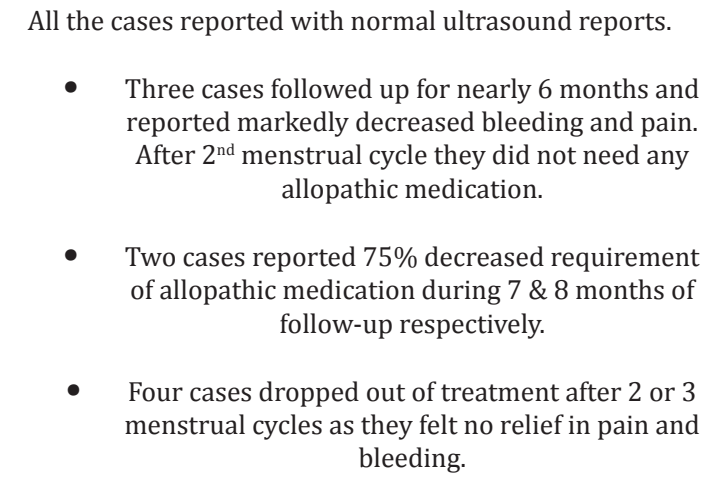 \\
\hline 24. & ASOM & $\begin{array}{c}30,200 \\
1000\end{array}$ & $\begin{array}{l}\text { Three doses } \\
\text { of any potency } \\
\text { prescribed: } \\
\text { each to be taken } \\
\text { every } 12 \text { hours }\end{array}$ & $12-24$ hours & $\begin{array}{l}\text { All the cases recovered within } 10 \text { days of treatment. } \\
\text { All the cases were prescribed three doses of } 30^{\text {th }} \text { potency } \\
\text { initially. } \\
\text { - Two cases recovered without further repetition of } \\
\text { medicine } \\
\text { - Seven cases were prescribed } 200^{\text {th }} \text { potency if pain \& } \\
\text { fever persisted on } 3^{\text {rd }} \text { day too. } \\
\text { - One case required } 1000^{\text {th }} \text { potency on } 7^{\text {th }} \text { day due to } \\
\text { persistent pain. }\end{array}$ \\
\hline 25. & Glue ear & 30,200 & $\begin{array}{l}\text { Three doses } \\
\text { of any potency } \\
\text { prescribed: } \\
\text { each to be taken } \\
\text { every } 12 \text { hours }\end{array}$ & 3-4 weeks & $\begin{array}{l}\text { Three cases followed up for nearly } 6 \text { months and } \\
\text { reported no discharge after } 2 \text { or } 3 \text { months of } \\
\text { medication. }\end{array}$ \\
\hline 26. & CSOM & 200 & $\begin{array}{l}\text { Three doses: } \\
\text { each to be taken } \\
\text { every } 12 \text { hours }\end{array}$ & 3-5 weeks & $\begin{array}{l}\text { All were diagnosed cases; two with perforation. } \\
\begin{array}{l}\text { - Three cases followed up for nearly } 6 \text { months and } \\
\text { reported no discharge after } 2 \text { or } 3 \text { months of } \\
\text { medication. }\end{array} \\
\text { - One case reported } 75 \% \text { reduction in discharge and } \\
\text { then dropped out citing slow improvement. }\end{array}$ \\
\hline 27. & Thyrotoxicosis & 200,1000 & $\begin{array}{l}\text { Three doses } \\
\text { of any potency } \\
\text { prescribed: } \\
\text { each to be taken } \\
\text { every } 12 \text { hours }\end{array}$ & -- & $\begin{array}{l}\text { - One girl diagnosed with graves disease and } \\
\text { post surgery advised radioactive iodine therapy. } \\
\text { After lachesis 200, reported over } 9 \text { months with } \\
\text { marked symptomatic improvement, improved } \\
\text { eye signs \& symptoms, weight gain, reduced } \\
\text { allopathic medication (neomercazole and ciplar). } \\
\text { Radioactive iodine therapy deferred by consulting } \\
\text { endocrinologist. } \\
\text { One lady reported symptomatic improvement over } \\
6 \text { months of follow-up. No change in allopathic } \\
\text { medication. }\end{array}$ \\
\hline
\end{tabular}




\begin{tabular}{|c|c|c|c|c|c|}
\hline 28. & Melasma & 200 & $\begin{array}{c}\text { Three doses: } \\
\text { each to be taken } \\
\text { every } 12 \text { hours }\end{array}$ & 6-8 weeks & $\begin{array}{l}\text { All the cases were asked to refrain from using any medicated/ } \\
\text { herbal face product. } \\
\text { - In two cases (one man) pigmentation cleared up } \\
\text { within } 4 \text { months } \\
\text { Three cases followed up for nearly } 6 \text { months and } \\
\text { reported nearly } 75 \% \text { reduction in pigmentation. } \\
\text { Two cases reported } 30-40 \% \text { decreased } \\
\text { requirement of allopathic medication during } 4 \text { \& } 6 \\
\text { months of follow-up respectively. } \\
\text { Two cases dropped out of treatment after } 4 \text { follow- } \\
\text { ups due to village visit. }\end{array}$ \\
\hline 29. & $\begin{array}{l}\text { Carpal tunnel } \\
\text { syndrome }\end{array}$ & $\begin{array}{c}30,200 \\
1000\end{array}$ & $\begin{array}{l}\text { One dose of } \\
\text { each potency } \\
\text { on three } \\
\text { consecutive } \\
\text { mornings }\end{array}$ & -- & $\begin{array}{l}\text { One case improved completely within } 2 \text { months of } \\
\text { follow-up. } \\
\text { - Three cases dropped out of treatment after 4-6 } \\
\text { follow-ups citing slow improvement/ village visit. }\end{array}$ \\
\hline 30. & Dengue fever & 30,200 & $\begin{array}{l}\text { Three doses } \\
\text { of any potency } \\
\text { prescribed: } \\
\text { each to be taken } \\
\text { every } 12 \text { hours }\end{array}$ & 24-36 hours & $\begin{array}{l}\text { All consulted with platelet counts less than } 1 \text { lac; none got } \\
\text { dengue NS1 antigen, TLC or PCV tested citing financial } \\
\text { inability. } \\
\text { All cases were initially prescribed three doses of } 30^{\text {th }} \text { potency. } \\
200^{\text {th }} \text { potency was prescribed in each case on } 3^{\text {rd }}-4^{\text {th }} \text { day due to } \\
\text { persistent pains. } \\
\text { No case required anti-pyretic drug after homoeopathic } \\
\text { treatment. } \\
\text { All cases were afebrile by } 3^{\text {rd }} \text { day and by } 5^{\text {th }} \text { day, had no pains. } \\
\text { Only one case repeated platelet count on } 3^{\text {rd }} \text { and } 5^{\text {th }} \text { day, which } \\
\text { were } 1.2 \text { lacs and } 1.9 \text { lacs respectively. }\end{array}$ \\
\hline 31. & $\begin{array}{l}\text { Chikungunya } \\
\text { fever }\end{array}$ & 30,200 & $\begin{array}{l}\text { Three doses } \\
\text { of any potency } \\
\text { prescribed: } \\
\text { each to be taken } \\
\text { every } 12 \text { hours }\end{array}$ & 24-36 hours & $\begin{array}{l}\text { Only one came with chikungunya IgM antibody (pre- } \\
\text { tretament); others were diagnosed symptomatically. } \\
\text { All the cases were initially prescribed three doses of } 30^{\text {th }} \\
\text { potency. } 200^{\text {th }} \text { potency was prescribed in each case on } 3^{\text {rd }}-4^{\text {th }} \\
\text { day due to persistent pains. } \\
\text { No case required anti-pyretic drug after homoeopathic } \\
\text { treatment. } \\
\text { All cases were afebrile by } 3^{\text {rd }} \text { day and by } 6^{\text {th }} \text { day, had no } \\
\text { residual pains or symptoms. }\end{array}$ \\
\hline 32. & $\begin{array}{l}\text { Post chikungya } \\
\text { chronic arthritis }\end{array}$ & $\begin{array}{c}30,200 \\
1000\end{array}$ & $\begin{array}{l}\text { One dose of } \\
\text { each potency } \\
\text { on three } \\
\text { consecutive } \\
\text { mornings }\end{array}$ & 7 days & $\begin{array}{l}\text { She cited persistent pains after an episode of chikungunya } \\
\text { fever } 1 \text { year back (IgM positive). } \\
\text { Pains, stiffness and weakness disappeared within } 5 \text { weeks of } \\
\text { homoeopathic treatment. } \\
\text { CRP and IgM were not repeated by patient citing financial } \\
\text { inability. }\end{array}$ \\
\hline
\end{tabular}




\begin{tabular}{|c|c|c|c|c|c|}
\hline 33. & Anal fissure & 200 & $\begin{array}{l}\text { Three doses: } \\
\text { each to be taken } \\
\text { every } 12 \text { hours }\end{array}$ & $\begin{array}{l}24-48 \text { hours for } \\
\text { pain and/ or } \\
\text { bleeding to stop } \\
\text { Symptom free } \\
\text { within a week }\end{array}$ & $\begin{array}{l}\text { None of the patients could comply with the advise of sitz bath. } \\
\text { No local applications were allowed/ prescribed. } \\
\text { Three patients presented with acute anal fissure and became } \\
\text { symptom free within a week's time. } \\
\text { Three patients consulted for chronic anal fissure } \\
\text { o One remained symptom free after a week all } \\
\text { through her } 11 \text { months follow up. } \\
\text { o Two patients with symptomatic relief within a } \\
\text { week's time dropped out of treatment after } 6 \\
\text { follow-ups due to village visit. }\end{array}$ \\
\hline 34. & Hemorrhoids & 30,200 & $\begin{array}{l}\text { Three doses } \\
\text { of any potency } \\
\text { prescribed: } \\
\text { each to be taken } \\
\text { every } 12 \text { hours }\end{array}$ & $\begin{array}{l}24-48 \text { hours for } \\
\text { bleeding to stop }\end{array}$ & $\begin{array}{l}\text { Clinical history suggested that three patients were having } 1^{\text {st }} \\
\text { degree hemorrhoids and one } 2^{\text {nd }} \text { degree hemorrhoids. } \\
\text { - Three patients (including one with } 2^{\text {nd }} \text { degree } \\
\text { hemorrhoids) remained symptom free for 6-8 } \\
\text { months of follow-up. } \\
\text { - One patient, symptom free, dropped out after } 2 \\
\text { months of treatment without citing any reason }\end{array}$ \\
\hline 35. & Psoriasis & 30,200 & $\begin{array}{l}\text { Three doses } \\
\text { of any potency } \\
\text { prescribed: } \\
\text { each to be taken } \\
\text { every } 12 \text { hours }\end{array}$ & -- & $\begin{array}{l}\text { All the cases were having multiple lesions, and were asked to } \\
\text { discontinue all local applications (except coconut oil) during } \\
\text { the course of treatment. } \\
\text { All the cases have been followed up for 6-11 months and are } \\
\text { still under observation. } \\
\text { Lesions tend to clear up above downwards and } \\
\text { new lesions were seen to appear only distally, in } \\
\text { accordance with Hering's law. } \\
\text { No repetition of remedy in any case after initial } \\
\text { prescription }\end{array}$ \\
\hline 36. & $\begin{array}{c}\text { Epidermolysis } \\
\text { bullosa }\end{array}$ & 200 & One dose & -- & $\begin{array}{l}\text { Pre-diagnosed case at Safdarjung hospital with histo- } \\
\text { pathology reports and prescriptions. } \\
\text { Prescription was based on the intra-uterine history of her } \\
\text { mother's rivalry with her sister in law, both vying for her } \\
\text { father's attention, left sided onset of lesion and worsening of } \\
\text { her lesions after birth of younger sibling. } \\
\text { Lesions cleared out in accordance with Hering's law } \\
\text { and skin cleared in } 4 \text { months completely. } \\
\text { - No relapse over last } 6 \text { months. }\end{array}$ \\
\hline
\end{tabular}

\section{Discussion}

\section{Importance of polychrest remedies}

The identification of polychrest remedies in our materia medica is just another of Hahnemann's contribution to clinical homoeopathic practice. All those practitioners of homeopathy viz. Hering, Stapf, Gross, Hale etc., who strictly adhered to Hahnemann's guidelines while conducting drug provings, added to the treasury of polychrest remedies, which can be useful in multiple diseased conditions, both acute and chronic. The reality check is that none of the recently proved drugs can be added to the group.

\section{Prescribing on concomitants/ characteristic symptoms}

Prescribing on the basis of concomitants or characteristic symptoms forms the backbone of homeopathic practice. It was 
seen in all the 193 patients, who received Lachesis muta, that drug selection was not dependent on common pathological (pathognomonic) symptomatology, rather on non-diagnostic symptomatolgy. And, wherever, local symtomatology was considered, modalities guided the remedy selection. Common symptoms are therefore usually not of much use in homeopathic drug prescribing and homeopathic case taking should delineate all the characteristic features/ concomitant symptoms or modalities that shall guide the remedy selection.

\section{No specifics in homoeopathy}

Since the remedy selection is solely dependent on the nonpathognomonic symptoms of the case, it rules out the possibility of having absolute specific remedies for any diseased condition in homeopathy.

\section{Symptoms of mind and disposition are mostly observable signs}

Six symptoms of mind and disposition of Lachesis muta: intolerance of constriction around neck, insane jealousy, religious disposition, loquacity, sibling rivalry and suspicion, which were clinically verified, were mostly interpreted from close observation of patient's behavior or demeanor. Since these symptoms are observable signs by the attending physician, the importance

Table 5: Clinical sphere of Lachesis muta. of thorough case taking, with close monitoring of the patients expressions and gestures is vital for homoeopathic treatment [12].

\section{Homeopathy is effective for both acute and chronic diseased conditions}

Homeopathic treatment is effective for both the acute and chronic diseased conditions. This has especially been verified for the remedy Lachesis muta.

\section{Gauging the remedial response time}

It was strongly verified in various acute conditions, that the response to indicated homoeopathic remedy can be judged within a few hours.

\section{Clinical verification of symptoms}

Twenty-eight signs and symptoms of Lachesis muta, mentioned in the homoeopathic literature could be clinically verified. Out of these, nearly 17 were objective.

\section{Chalking the clinical sphere of a homoeopathic remedy}

At the PHC, the remedy was found to be useful in 36 diverse clinical conditions, some of which have been mentioned in full or part by Hering and Clarke and this may be seen below in Table 5 .

\begin{tabular}{|c|c|c|c|}
\hline S.No. & Name of DISEASE/ DIAGNOSIS & Hering's Guiding Symptoms & Clarke's Dictionary \\
\hline 1. & Bronchial asthma & $\checkmark$ & $\checkmark$ \\
\hline 2. & Migraine & $\begin{array}{c}\text { - (Headache, megrim, } \\
\text { cephalalgia, supraorbital } \\
\text { neuralgia are mentioned) }\end{array}$ & -(Headache is mentioned) \\
\hline 3. & $\begin{array}{l}\text { Upper respiratory tract infection with } \\
\text { fever }\end{array}$ & $\begin{array}{l}\text { - (Coryza, affections of nose, } \\
\text { scarlet fever, tonsillitis, throat } \\
\text { affection of are mentioned) }\end{array}$ & $\begin{array}{l}\text { - (Sore throat, scarlatina and } \\
\text { hay fever are mentioned) }\end{array}$ \\
\hline 4. & $\begin{array}{l}\text { Non-specific cervical lymphadenitis (in } \\
\text { children) }\end{array}$ & - & - \\
\hline 5. & Warts & - & $\checkmark$ \\
\hline 6. & Tinea capitis & $\begin{array}{l}\text { - (Skin affection and itch are } \\
\text { mentioned) }\end{array}$ & - \\
\hline 7. & Tinea cruris & $\begin{array}{c}\text { - (Skin affection, itch, eruptions } \\
\text { on arms \& abdomen, eczema } \\
\text { are mentioned) }\end{array}$ & - \\
\hline 8. & Vitiligo & - & - \\
\hline 9. & Acne vulgaris & $\begin{array}{l}\text { - (Skin affections and pustular } \\
\text { eruptions are mentioned) }\end{array}$ & - \\
\hline 10. & Seborrhoeic dermatitis & $\begin{array}{l}\text { - (Skin affection, eczema and } \\
\text { itch are mentioned) }\end{array}$ & - \\
\hline 11. & Amoebic colitis & $\begin{array}{l}\text { - (Diarrhoea, croupous } \\
\text { enteritis, typhilitis and } \\
\text { dysentry are mentioned) }\end{array}$ & $\begin{array}{l}\text { - (Caecum, affections of is } \\
\text { found })\end{array}$ \\
\hline 12. & Irritable bowel syndrome & $\begin{array}{c}\text { - (Dyspepsia, diarrhoea, } \\
\text { constipation and dysentry are } \\
\text { mentioned) }\end{array}$ & $\begin{array}{l}\text { - (Dyspepsia, flatulence are } \\
\text { mentioned) }\end{array}$ \\
\hline
\end{tabular}




\begin{tabular}{|c|c|c|c|}
\hline 13. & Hypothyroidism & - & - \\
\hline 14. & Diabetes mellitus & - & - \\
\hline 15. & Hypertension & $\begin{array}{c}\text { - (Retinitis apoplectica is } \\
\text { found) }\end{array}$ & - (Apoplexy is found) \\
\hline 16. & Rheumatoid arthritis & $\begin{array}{c}\text { - (Pain below knee, pain in } \\
\text { joints are found) }\end{array}$ & - \\
\hline 17. & Alopecia areata & - & - \\
\hline 18. & Molluscum contagiosum & - & - \\
\hline 19. & COPD & $\begin{array}{c}\text { - (bronchitis, bronchial } \\
\text { catarrh, respiratory affections, } \\
\text { dyspnoea, asthmatic cough, } \\
\text { lung affections etc are } \\
\text { mentioned) }\end{array}$ & - (Cough, asthma, \\
\hline 20. & LRTI with fever & $\begin{array}{l}\text { - (bronchitis, respiratory } \\
\text { affections, chest/lung } \\
\text { affections are mentioned) }\end{array}$ & - (Pneumonia is mentioned) \\
\hline 21. & Ovarian cyst & $\begin{array}{c}\text { - (Ovaritis and ovarian tumor } \\
\text { are mentioned) }\end{array}$ & $\begin{array}{c}\text { - (Ovaries, affections of, is } \\
\text { mentioned) }\end{array}$ \\
\hline 22. & Uterine fibroids & $\begin{array}{l}\text { - (Uterine tumors are } \\
\text { mentioned) }\end{array}$ & - (Tumors are mentioned) \\
\hline 23. & DUB & - & $\begin{array}{c}\text { - (Hemorrahges are } \\
\text { mentioned) }\end{array}$ \\
\hline 24. & ASOM & $\begin{array}{c}\text { - (Affection of ear is } \\
\text { mentioned) }\end{array}$ & $\begin{array}{l}\text { - (Ear, otorrhoea are } \\
\text { mentioned })\end{array}$ \\
\hline 25. & Glue ear & $\begin{array}{c}\text { - (Affection of ear is } \\
\text { mentioned) }\end{array}$ & $\begin{array}{l}\text { - (Ear, otorrhoea are } \\
\text { mentioned) }\end{array}$ \\
\hline 26. & CSOM & $\begin{array}{l}\text { - (Affection of ear, partial } \\
\text { deafness are mentioned) }\end{array}$ & $\begin{array}{c}\text { - (Ear noises in, otorrhoea are } \\
\text { mentioned) }\end{array}$ \\
\hline 27. & Thyrotoxicosis & - & - \\
\hline 28. & Melasma & - & - \\
\hline 29. & Carpal tunnel syndrome & - & - \\
\hline 30. & Dengue fever & - & - \\
\hline 31. & Chikungunya fever & - & - \\
\hline 32. & Post chikungunya chronic arthritis & $\begin{array}{l}\text { - (Pain below knee, pain in } \\
\text { joints are found) }\end{array}$ & - \\
\hline 33. & Anal fissure & $\begin{array}{l}\text { - (Affections of anus and } \\
\text { rectum are mentioned) }\end{array}$ & - \\
\hline 34. & Hemorrhoids & $\checkmark$ & $\checkmark$ \\
\hline 35. & Psoriasis & - (Skin affections is mentioned) & - \\
\hline 36. & Epidermolysis bullosa & $\begin{array}{l}\text { - (Skin affection, pustular } \\
\text { eruption are mentioned) }\end{array}$ & - \\
\hline
\end{tabular}

\section{Limitations of study}

All the patients who received Lachesis muta could only be observed for clinical improvement. Only a few of them could be documented properly with investigation reports due to poor financial status of patients and limited resources.

\section{Possibility of a bias in study}

Since the entire data collection and review was done single handedly by the author, there may be a possibility of confirmation bias. This fact was also pointed out during the recently concluded 3rd conference organized by Homeopathy Research Institute, where this paper was presented. Simultaneously, another researcher pointed out that if we move from the two basic observations viz. intolerance of constriction around neck and fine tremors on tongue, then we are already biased about these symptoms as guiding symptoms of the remedy and we have not regarded the possibility of prescribing the remedy in 
those patients who do not have it or manifest an opposite state. However, to this objection, I can only respond by saying that just like it is impossible to conceive of an elephant without a trunk, it is similarly impossible to conceive of a patient of Lachesis muta without these two cardinal symptoms.

\section{Conclusion}

The concept of polychrest remedy is individual to homoeopathy alone. We have attempted to gauge the vast clinical applicability of one such polychrest, Lachesis muta in the PHC set-up over a year.

It has been many years since Hering, Allen or Clarke published their materia medica mentioning the clinical conditions responding to each remedy by that time, many of which are today found to be obsolete and requiring a scientific appraisal. This work is a first attempt to clinically (scientifically) verify some of its symptomatology, identify the clinical sphere amenable to such a polychrest remedy in the current scenario, with due regards to the textbooks of homoeopathic materia medica of these stalwarts, and gauge the posology and remedy response time as well as therapeutic outcome.

Further multi-centric studies with more scientific rigour can validate as well as clinically verify the symptomatology of Lachesis muta by the use of Bayesian statistics.

\section{References}

1. Hahnemann S (1999) Materia Medica Pura-Vol II. B Jain Publishers (P) Ltd, India, pp. 345.
2. Hering C (1971) The Guiding Symptoms of our Materia Medica-Vol VI. B Jain Publishers, India, pp. 559.

3. Hering C (1971) The Guiding Symptoms of our Materia Medica-Vol VI. B Jain Publishers, India, pp. 559-562.

4. Hering C (1971) The Guiding Symptoms of our Materia Medica-Vol VI. B Jain Publishers, India, Pp. 559-650.

5. Allen TF (2011) The Encyclopedia of Pure Materia Medica-Vol V, $6^{\text {th }}$ impression. B Jain Publishers (P) Ltd, India, pp. 432-471.

6. Clarke JH (1999) A Dictionary of Practical Materia Medica-Vol II. B Jain Publishers (P) Ltd. India, pp. 210-227.

7. Roberts HA (2004) The Principles and Art of Cure by Homeopathy. Indian Books \& Periodicals Publishers, New Delhi, India, pp. 144149.

8. Allen HC (1967) Keynotes and characteristics with comparisons of some of the leading remedies of the materia medica. Roy Publishing House, Calcutta, India, pp.159-162.

9. Hering C (1971) The Guiding Symptoms of our Materia Medica-Vol VI. B Jain Publishers (P) Ltd, India, pp. 645.

10. Barthel H (1993) Synthetic Repertory-Vol I: Psychic symptoms. (4 ${ }^{\text {th }}$ edn), B Jain Publishers (P) Ltd, India, pp. 523.

11. Barthel H (1999) Synthetic Repertory-Vol III: Sleep, Dreams, Sexuality. ( $4^{\text {th }}$ edn), B Jain Publishers (P) Ltd, India, pp. 350.

12. Wadhwani G (2013) Uterine Fibroma: A case cured by Homeopathy. AJHM Autumn 106(3): 120-124. 